EchoGéo

ECHOGEO

55 | 2021

Espace et parentalité

\title{
Parents voyageurs. Croisières familiales au long cours
}

Anne Gaugue

\section{(2) OpenEdition}

1 Journals

Édition électronique

URL : https://journals.openedition.org/echogeo/20861

DOI : $10.4000 /$ echogeo.20861

ISSN : 1963-1197

Éditeur

Pôle de recherche pour l'organisation et la diffusion de l'information géographique (CNRS UMR 8586)

Référence électronique

Anne Gaugue, "Parents voyageurs. Croisières familiales au long cours », EchoGéo [En ligne], 55 | 2021, mis en ligne le 31 mars 2021, consulté le 10 août 2021. URL : http://journals.openedition.org/ echogeo/20861; DOI : https://doi.org/10.4000/echogeo.20861

Ce document a été généré automatiquement le 10 août 2021.

EchoGéo est mis à disposition selon les termes de la licence Creative Commons Attribution - Pas d'Utilisation Commerciale - Pas de Modification 4.0 International (CC BY-NC-ND) 


\title{
Parents voyageurs. Croisières familiales au long cours
}

\author{
Anne Gaugue
}

1 Partir en famille, pendant plusieurs mois ou plusieurs années, et effectuer un tour, du monde ou d'un continent, tel est le choix des parents voyageurs. C'est à partir de l'exemple des plaisanciers au long cours que nous proposons d'aborder la géographie de ces parents.

2 L'itinérance des plaisanciers au long cours à la recherche d'un mode de vie axé sur le plaisir et le bien être est une mobilité à finalité récréative qui relève des lifestyle migrations ainsi que des lifestyle mobilities. La notion de lifestyle migrations, proposée par M. Benson et K. O'Reilly, désigne « the spatial mobility of relatively affluent individuals of all ages, moving either part-time or full-time to places that, for various reasons, signify, for the migrant, a better quality of life $»^{1}$ (Benson et O'Reilly, 2009, p. 609). La croisière au long cours s'apparente à ces lifestyle migrations ou migrations d'agrément, car c'est bien la recherche d'une meilleure qualité de vie qui pousse les plaisanciers au long cours à habiter en mer. Mais en choisissant l'itinérance, les plaisanciers répondent à cette quête d'une autre façon que les populations analysées dans le cadre des life style migrations qui privilégient l'étude des mobilités d'un lieu A à un lieu B. Pour désigner les mobilités relevant de "on-going semi-permanent moves of varying durations ${ }^{2}$, S. Cohen, T. Duncan et M. Thulemark proposent la notion de lifestyle mobilities (2016, p. 4). Les articles rassemblés par ces auteurs dans l'ouvrage collectif Lifestyle Mobilities: Intersections of Travel, Leisure and Migration illustrent la diversité de ces modes de vie mobilitaires, des artistes aux employés d'un cirque, des autostoppeurs aux plaisanciers au long cours.

Pour désigner le fait de parcourir une partie ou l'ensemble du monde pour son plaisir, nous proposons la notion d'itinérance récréative. Certaines de ces itinérances sont bien connues, des backpackers à ceux qui, résidant dans un camping-car, vivent sur la route. Les travaux mettent en évidence la prédominance de deux classes d'âge chez ces itinérants : les backpackers sont majoritairement de jeunes adultes qui n'ont pas encore d'enfants (Le Bigot, 2017) et les camping-caristes itinérants des retraités dont les 
enfants sont devenus autonomes (Forget, 2012, Vacher, 2004). Cependant, l'itinérance récréative ne saurait se réduire à ces deux tranches d'âge. Le terme de flashpackers, proposé en 2010 par J. Jarvis et V. Peel, permet de rendre compte de l'émergence de «backpackers plus âgés et déjà entrés dans la vie active » (Le Bigot, 2017, p. 272). Les couples voyageant avec enfants, certes minoritaires, sont présents sur les routes. Dans son ouvrage Vivre sur la route. Les nouveaux nomades nord-américains, C. Forget (2012) mentionne des familles voyageant à bord de leur camping-car. Un certain nombre de sites internet proposent des conseils aux parents tentés par l'aventure et de nombreux blogs relatent les péripéties de familles en chemin. Le site "Voyager en famille ${ }^{3}$ renvoie ainsi sur plus de 300 blogs de parents voyageurs partis plusieurs mois et qui se déplacent de lieu en lieu, essentiellement en avion ou en camping-car mais également à vélo ou en voilier.

4 C'est à partir de l'exemple des plaisanciers au long cours que nous proposons d'aborder la géographie des parents voyageurs, ceux qui durant au moins une année et souvent bien davantage naviguent d'escale en escale avec leurs enfants. Il y aurait entre 8 et 10000 voiliers de grande plaisance naviguant autour du monde (Cornell, 2017). À partir des enquêtes effectuées par J. Cornell, complétées par des études menées par certaines autorités portuaires, on peut estimer qu'environ $25 \%$ des équipages sont constitués de parents naviguant avec leurs enfants (Gaugue, 2020).

5 Dans le cadre de cette recherche, nous avons constitué un corpus de quarante-neuf plaisanciers adultes, en grande majorité français, qui ont effectué une croisière d'au moins une année avec leurs enfants, et ce depuis 1980 - date à laquelle les croisières familiales se diffusent. La plupart de ces parents plaisanciers ont - comme les autres plaisanciers - suivi des études supérieures et font partie des catégories sociales privilégiées, notamment par leur niveau de formation. La durée de leur itinérance est variable : dix-neuf adultes ont navigué entre un et deux ans, vingt-quatre entre trois et cinq ans et six plus de dix ans, avec parfois des interruptions de quelques années à terre. Les routes de grande croisière s'établissant en fonction des vents et courants dominants, les plaisanciers suivent sensiblement les mêmes itinéraires et privilégient les mêmes lieux d'escale. Ceux partis pour un ou deux ans effectuent un tour de l'Atlantique, des côtes françaises aux Antilles; au-delà de cette durée, les plaisanciers font le plus souvent un tour du monde. Pour financer leur périple, ceux qui naviguent moins de deux ans ont économisé la somme nécessaire avant le départ et ceux qui vivent à bord plusieurs années effectuent de longues escales afin de travailler pour renflouer la caisse de bord.

6 La moitié de ces récits de grande croisière a été recueillie sur des blogs ${ }^{4}$, l'autre moitié a fait l'objet de publications : livres et surtout articles, parus dans la presse spécialisée. Nous avons par ailleurs mené, en novembre 2008, quatre entretiens auprès de parents voyageurs, à Las Palmas (Canaries), port de départ avant la traversée de l'Atlantique. Ces différentes sources n'offrent pas la même vision de la grande croisière. Dans les articles et livres, publiés à l'issue de la grande croisière, c'est le plus souvent une image idyllique de la vie en mer qui prévaut. Les blogs, écrits au cours du voyage, donnent la possibilité d'avoir accès à un moment de vie, à un instantané de pratiques et de représentations et laissent davantage entrevoir les contraintes au quotidien. Dans ces récits de vie, nous avons été attentive à la place des enfants - lors de la définition du projet et durant la croisière, ainsi qu'à la manière dont leur présence marquait le rythme du voyage. 
7 La grande croisière est un mode de vie alternatif choisi par les parents qui tentent de concilier leur désir de vivre autrement sans pour autant marginaliser leurs enfants. Dès lors, comment être, tout à la fois, parents et voyageurs ? Dans quelle mesure la fonction parentale oriente-t-ell les spatialités et joue-t-elle un rôle sur le projet de voyage ainsi que sur le choix des lieux d'escales et sur les pratiques qui s'y déroulent? Dans un premier temps, nous analyserons la place de l'enfant dans les motivations parentales, puis nous verrons les contraintes que font peser ces derniers sur le rythme et l'itinéraire de la grande croisière avant d'aborder les éventuels apports du voyage pour la famille, lors des escales ou durant les navigations.

\section{Au centre du projet de voyage, les enfants}

8 L'itinérance autour du monde en voilier répond à un projet parental qui, dans les témoignages des plaisanciers, est présenté comme une manière de concilier tout à la fois le désir de passer du temps avec ses enfants et de leur faire découvrir d'autres réalités que celles expérimentées dans leur pays d'origine.

Les cadences du rythme urbain ont pesé lourd dans la décision de B. Troublé de prendre une année sabbatique : «À Paris, on ne voit pas ses enfants. Pourrait-on les connaître en rentrant chaque soir à 21 heures alors qu'ils dorment déjà ? " (cité par Gourmelen 1997, p. 94). Dany et René ont embarqué avec leurs quatre enfants pour un tour du monde de cinq ans afin «de faire un temps d'arrêt, pour être en famille et voir le monde, car la vie va tellement vite, on ne voit pas les enfants grandir » (entretien, Las Palmas). Le voyage en famille participe d'une stratégie éducative et doit permettre aux enfants d'acquérir certaines compétences internationales, de l'apprentissage de langues étrangères à la capacité de s'adapter à l'altérité. La majorité des parents plaisanciers insistent sur la dimension pédagogique de la grande croisière, à l'image des Le Gall qui ont souhaité "vivre avec les enfants ailleurs et autrement. Leur faire rencontrer d'autres gens, d'autres modes de vie, d'autres cultures et religions » (Le Gall, 2003-2004). Depuis le Grand Tour, qui menait les jeunes aristocrates britanniques vers l'Italie, l'itinérance récréative a une forte dimension éducative et participe à la «construction des individus» (Équipe MIT, 2011, p. 41). La grande croisière s'inscrit dans cette filiation, l'ouverture à d'autres possibles permettant aux enfants de s'enrichir. Cependant, contrairement au Grand Tour et autres voyages initiatiques, cette démarche est, dans le cas des grandes croisières, non pas laissée à l'initiative individuelle de jeunes adultes mais est décidée et encadrée par des parents d'enfants non encore entrés dans l'adolescence.

L'âge des enfants est un critère essentiel pour déterminer la date de départ et la durée de la croisière au long cours. La grande majorité des enfants relèvent de l'école élémentaire, et si quelques très jeunes enfants embarquent sur des voiliers de grande croisière, les collégiens et lycéens sont quasiment absents. Ainsi, Olivier et Daphné ont attendu que leurs filles «soient assez grandes pour se souvenir du voyage mais pas encore adolescentes pour ne pas nous détester de les emmener en voyage loin de leurs amis » (Olivier et Daphné, 2010). À bord de Catmousses, Dany et René ont largué les amarres pour une circumnavigation de cinq ans lorsque leurs quatre enfants, alors âgés de 10, 8,7 et 5 ans, étaient « au bon âge pour partir : ils se souviendront du voyage et ils ne sont pas encore adolescents, à un âge où ils préfèreront ne plus être avec nous " (entretien, Las Palmas). 
11 Les plaisanciers considèrent que la grande croisière ne convient pas pour des adolescents. «L'entrée au collège marque une rupture dans les stratégies scolaires des parents » relèvent J-Y. Authier et S. Lehman-Frisch (2012, p. 63) dans un article sur la place des enfants dans des quartiers gentrifiés de Paris et San Francisco. Un constat similaire ressort de l'analyse des parents voyageurs : alors que l'encadrement scolaire en primaire ne constitue pas un frein à l'itinérance maritime en famille, les études à partir du collège sont considérées comme incompatibles avec ce mode de vie, car elles représentent, aux yeux des parents, un enjeu important pour l'avenir de leur enfant, et ils ne se sentent pas toujours capables de remplacer les enseignants. Après avoir vécu trois ans en voilier avec leur fils, Michel et Annie renoncent à l'itinérance maritime lorsque ce dernier entre en $6^{\text {ème }}$ et attendent qu'il ait acquis son indépendance pour retourner habiter en mer (entretien, Las Palmas). Par ailleurs, les parents craignent qu'une vie familiale se déroulant dans le huis clos d'un voilier ne convienne pas à un adolescent. C. Mailhot et D. Manny ont navigué six ans autour du monde avec leurs quatre enfants, Évangeline, leur fille aînée, ayant alors onze ans. Au bout de quatre ans de voyage, C. Mailhot se « rend compte qu'octobre 1986 représentait une date de départ limite pour Évangeline. Serions-nous partis deux ou trois ans plus tard, que notre vie de clan aurait connu davantage de soubresauts » (Mailhot et Manny, 2003, p. 171).

Des liens structurants apparaissent ainsi entre temporalités et spatialités familiales, l'itinérance récréative étant liée à l'âge des enfants. Lorsque les enfants des plaisanciers au long cours deviennent adolescents, leurs parents renoncent à la mobilité pour un mode de vie plus classique. L'influence de la scolarité de l'enfant sur le choix de vie des parents se retrouve dans d'autres mobilités d'agrément, comme en témoigne l'exemple des Occidentaux ayant élu résidence à Vârânasî (Inde) : " a crucial moment in deciding whether to stay in Vârânasî or not is actually not the birth of a child but when the child reaches school age $»^{5}$ (Korpela, 2009, p. 179), les parents préférant un établissement occidental aux structures scolaires locales.

13 Dans les témoignages de parents plaisanciers, le projet de grande croisière apparait comme étant centré autour de l'enfant, alors que ceux qui naviguent sans enfants avancent d'autres motifs, de la recherche d'une vie proche de la nature à la liberté que le voilier procure. Étudiant les familles occidentales vivant entre Goa et leur pays d'origine, Maria Korpela souligne que «the parents often emphasised that living there was of benefit to their children, with many of them seeming to explain their lifestyle by claiming that it was better for their children $»^{6}(2014$, p. 94). En insistant sur l'importance du voyage dans la construction de leurs enfants, les parents plaisanciers justifient également un choix qui est d'abord le leur, et l'aspect pédagogique du voyage est avancé pour légitimer le fait d'imposer à leur progéniture un mode de vie alternative... alors que le rêve de grand voyage maritime est d'abord celui du père.

Si la décision de partir est prise par les deux parents, elle résulte le plus souvent d'un compromis au sein du couple. Plus compétents que leurs conjointes en matière de navigation et aspirant à de longues navigations hauturières, ce sont les hommes qui sont à l'origine du projet. Les mères sont plus hésitantes - attirées par l'aspect voyage et découverte de la grande croisière, leurs craintes concernent les traversées océaniques et la gestion des enfants à bord. C'est après avoir négocié et obtenu satisfaction sur les éléments de confort et de sécurité qu'elles acceptent de suivre leur compagnon dans cette aventure familiale. Quant aux enfants, ils ne sont pas consultés et sont contraints de suivre leurs parents alors même que certains expriment leur 
appréhension. "On ne va pas faire des choses de la vraie vie ", s'inquiète Anna, neuf ans, avant son départ pour une croisière d'un an autour de l'Atlantique (Le Gall, 2003-2004. Embarquer de jeunes enfants est une manière d'éviter tout contestation de leur part. Certes, comme l'affirment les parents plaisanciers, il n'est pas aisé de suivre une scolarité au collège ou au lycée à distance, certes l'adolescent aime à s'extraire du cercle familial. Mais un adolescent est également plus à même qu'un enfant plus jeune de contester le choix de vie de ses parents et, au cours du voyage, la présence d'un équipier potentiellement rétif à ce mode de vie pèsera sur l'ambiance du bord. Les parents valorisent l'expérience de la croisière pour les enfants alors même que ce projet permet d'abord aux parents, et notamment au père, d'assouvir leurs rêves.

\section{Le voyage au rythme de la scolarité et de la sociabilité des enfants}

Pour préserver leurs enfants des éventuels aspects négatifs de ce mode de vie et faciliter leur retour à une vie terrestre et sédentaire, les parents adaptent le rythme et l'itinéraire de leur croisière à la scolarité ainsi qu'à la socialisation de ces derniers.

Alors que les adultes choisissent de s'offrir une pause sabbatique ou de ne travailler que ponctuellement et uniquement lorsque la nécessité s'en fait sentir, les parents constituant notre corpus n'envisagent pas de dispenser leurs enfants des contraintes scolaires et leur imposent un rythme de travail régulier. Mag et Bernard Perret ont navigué trois ans avec leurs trois enfants et ont tenu «à ce qu'ils suivent un régime scolaire normal, pour qu'ils puissent se réinsérer plus facilement le jour où nous reviendrons » (Perret, 1984, p. 32). Durant treize ans, les Maheu ont sillonné les océans du monde avec leurs deux enfants. Dès que ceux-ci sont en âge d'être scolarisés, la « contrainte des cours [devient] l'axe central de notre vie à bord. Le tourisme vient en second " (Maheu, 1989-2002). En escales, la matinée est généralement consacrée aux devoirs, l'après-midi étant réservé aux activités récréatives, plage ou visites. Si les parents voyageurs ne reproduisent pas à bord le rythme quotidien des établissements scolaires, ils respectent en revanche les rythmes hebdomadaires et annuels et accordent à leurs enfants la pause du week-end et des vacances.

Certains parents optent pour des cours par correspondance type CNED, d'autres suivent le programme en s'appuyant sur des manuels. Dans tous les cas, ils encadrent la scolarité de leurs enfants et la confusion des rôles entre parents et enseignants n'est pas toujours aisée. À bord de Loren, c'est Constance qui fait classe à ses trois enfants. «Le nombre des enfants et le double statut de mère et d'institutrice créent souvent des problèmes : les enfants refusent de travailler, négocient, se disputent... c'est souvent épuisant de les faire travailler» (de Montbron, 1996-1998). Pour résoudre cette difficulté, les parents utilisent les ressources du lieu d'escale et inscrivent les enfants à l'école locale, même pour quelques jours. À La Rochelle, les quatre enfants de Dany et René sont accueillis un mois à l'école primaire et les deux garçons des Meffre sont scolarisés une seule journée dans l'école de Pitcairn. Pour les parents, l'enjeu de ces très courts séjours dans les écoles locales n'est pas le niveau scolaire mais bien la possibilité pour leurs enfants d'être en contact avec des camarades de leur âge tout en se confrontant à l'altérité. Par ailleurs « cela nous libère bien » reconnaît M. Meffre (1997, p. 130) et envoyer ses enfants dans l'établissement scolaire du lieu d'escale permet de 
rompre le huis clos familial, parents et enfants bénéficiant alors d'un temps et d'un espace à eux.

Lorsque la croisière se déroule sur plusieurs années, certains parents choisissent de faire une escale prolongée dans un même lieu afin d'y scolariser leurs enfants, le choix du lieu étant alors directement lié au projet éducatif. C'est en Australie que René et Fabienne Ortmans décident de faire une escale de plusieurs mois pour que leur fils Anthony, alors âgé de 13 ans, passe une année scolaire dans un collège australien de façon à apprendre l'anglais. Dans la plupart des cas, les longues escales sont également motivées par la nécessité de travailler pour financer la poursuite du voyage. Dès lors, les plaisanciers français privilégient les collectivités et départements d'Outre-mer qui offrent aux parents la possibilité légale de travailler et de scolariser leurs enfants. "Le niveau scolaire est faible " constate Jean-Michel Maheu en Nouvelle Calédonie, mais l'objectif n'est pas de choisir l'établissement en fonction de ses résultats mais de permettre aux parents de travailler et aux enfants d'avoir une vie sociale. C'est à Saint Pierre et Miquelon que les de Neuville jettent l'ancre durant une année scolaire, pause appréciée par leur fils de treize ans : «J'ai repris le chemin de la vraie école, ravi de pouvoir faire une petite pause après une année de voyage. Je n'étais pas fâché de me retrouver pendant un assez long moment avec des gens de mon âge » (de Neuville, 1995, p. 92).

L'habiter itinérant suppose des départs toujours renouvelés ce qui n'est pas aisé pour les enfants qui doivent alors rompre les liens avec leurs amis. Au départ de Tamatave, Évangeline note dans son journal de bord: "J'ai des larmes dans les yeux encore une fois. C'est dur de partir quand on se fait des amis » (Mailhot et Manny, 2003, p. 203). Et l'absence de camarades de jeux pour leurs enfants pèse sur le moral des parents. Dans le "cadre enchanteur» du mouillage de Tongatapu (Tonga), Olivier et Daphné (2010-2020) notent qu'il « ne manque qu'un seul ingrédient: des amis pour les filles». Les parents sont attentifs à cette dimension qu'ils considèrent comme essentielle au bien-être de leurs enfants et n'hésitent pas à modifier leur programme pour leur permettre de rester avec leurs amis. Les Gaussen retardent leur départ de MarieGalante pour que leur fils de 7 ans puisse honorer une invitation d'un de ses camarades et pour leur première escale dans les îles de Las Perlas, Olivier et Daphné choisissent "celle où leurs amis de Maïa veulent brièvement s'arrêter. [...] Pour les enfants, l'objectif est de maximiser le temps avec leurs amis» (Olivier et Daphné, 2010-2020).

Par l'intermédiaire des enfants, des liens se nouent entre parents. En Martinique, les Le Gall apprécient de rencontrer trois équipages français voyageant en famille: « l'ambiance est très sympa, ça parle CNED, bouquins, pêche et évidemment pour nous les apéros se multiplient » (Le Gall, 2003-2004). À l'instar de certains quartiers, se développent dans les ports de plaisance ou les mouillages, des «sociabilités de famille, renforcées par la proximité socio-spatiale et par les amitiés croisées des enfants et des parents » (Authier et Lehman-Frisch, 2012, p. 69). Dans ces "villages flottants » que sont les ports de plaisance ou les mouillages, la sociabilité dominante relève indéniablement de l'entre-soi, caractéristique commune à la plupart des itinérances récréatives, des backpackers (Cohen, 2003 ; Le Bigot, 2017) aux camping caristes (Forget, 2012) et plus généralement aux migrations d'agrément. Ainsi, M. Korpela souligne l'importance de l'entre-soi chez les Occidentaux résidant à temps partiel en Inde: « lifestyle migrant families seldom socialise with local Indian families» (2014, p. 96). 
Dès lors, «children are not socialised into the local surrounding cultures but into "a Western lifestyle" $»^{7}$ (Korpela, 2014, p. 102).

\section{Ouverture au monde et huis clos familial}

Lorsque les devoirs scolaires sont terminés, place est faite à l'expérience et à la découverte de réalités autres permettant aux enfants de se construire. Dans sa thèse portant sur l'habitabilité des voiliers de plaisance, S. Brulé-Josso souligne que les "quelques ouvrages concernant la présence de jeunes enfants à bord, publiés à la fin des années soixante-dix et début quatre-vingt, reprennent les thématiques de l'éducation nouvelle afin d'asseoir la légitimité de ce type de navigation» (2010, p. 979). Aujourd'hui encore, les parents plaisanciers sont attentifs à ce que leurs enfants deviennent des «acteurs actifs du processus d'acquisition des connaissances » (BruléJosso, 2010, p. 979), suivant en cela les préceptes de l'éducation nouvelle qui considère qu'«aller de la liberté de principe à la liberté réalisée - que l'on pourrait appeler autonomie - devrait être un principe directeur $»^{8}$.

Le voyage est supposé permettre aux enfants de s'ouvrir au monde et les parents valorisent, dans leurs récits, cette expérience qui permet de se confronter à l'altérité ou de gagner en autonomie.

Dans les escales à fort différentiel d'altérité, la confrontation des enfants avec la pauvreté acquiert une valeur pédagogique. Au Cap Vert, "nos enfants paraissent surpris de ce qu'ils voient, mais c'est aussi une approche intéressante pour eux » estime Le Gall (2003-2004). C. Mailhot se réjouit que ses enfants aient pu voir des Sri Lankais venir fouiller leurs poubelles: "Pour les mettre face à une réalité, on ne pouvait espérer mieux. En quinze secondes, nos enfants venaient de comprendre l'essentiel » (Mailhot et Manny, 2003, p. 87). En revanche, la confrontation avec la pauvreté n'est pas toujours appréciée des enfants comme en témoigne Sandrine, la fille de C. Mailhot, qui écrit garder un "mauvais souvenir des villes de pays pauvres, comme Colombo au Sri Lanka ou Antanarivo à Madagascar " (Mailhot et Manny, 2003, p. 399).

Lorsque le voilier est à quai dans une marina, endroit clos sécurisé, ou au mouillage dans une île déserte ou peu peuplée, les enfants vont et viennent à leur guise et retrouvent d'autres enfants de bateau, loin du regard des parents. Analysant l'impact des activités extra scolaires sur la géographie des parents, S. Holloway et H. PimlottWilson soulignent que " chauffeuring children between activities is a labor-intensive process for the parents $»^{9}(2014$, p. 622$)$. À bord d'un voilier de plaisance, rien de tel et même lorsque ce dernier est à l'ancre au mouillage, ce qui implique d'utiliser une embarcation auxiliaire ou annexe pour rejoindre la terre, les enfants développent des compétences mobilitaires pour pouvoir se déplacer sans avoir recours à leurs parents. Ainsi, les filles d'Olivier et Daphné «conduisent seules une annexe à 10 ans environ. Cela permet d'avoir une première expérience tôt de la conduite d'un engin motorisé et d'en appréhender les risques » (Olivier et Daphné, 2010-2020).

Lors des grandes croisières, les marinas ainsi que les motu polynésiens et autres îlots tropicaux faiblement peuplés sont des espaces où l'encadrement parental se relâche. Les enfants, responsabilisés, gagnent dès lors en autonomie. Ces lieux d'escale présentent des caractéristiques similaires à certains quartiers urbains analysés par C. Rivière: l'absence de circulation automobile et l'exercice d'une surveillance collective permet de les "ériger en arènes sécurisées d'entraînement à l'autonomie " 
(Rivière, 2017, p. 178). Lors de ces escales, les autres plaisanciers au long cours, mais également les résidents locaux, assurent la fonction d'"yeux de la rue», selon l'expression de Jane Jacobs (cité par Rivière, 2017, p. 182). Sur le motu d'Hirifa à Fakarava (Tuamotu), Gek, gardien d'un restaurant, «s'est montré très gentil avec les enfants, à qui il offrait des cocos fraîches. Il leur a aussi installé une balançoire sous un cocotier sur la plage. Dans un tel environnement, nous pouvons faire nos activités l'esprit tranquille» (Olivier et Daphné, 2010-2020). M. Mikkelsen et B. Blichfeldt soulignent que « a successful holidays provides freedom from family commitments to allow all the family membres to enjoy their holidays both together and apart $»^{10}(2018$, p. 100). Des enfants autonomes cela signifie également des parents qui ont du temps pour eux... et ces occasions de séparations familiales sont appréciées par les deux parties.

La liberté dont les enfants bénéficient lors de certaines escales n'est pas de mise lors des navigations. Habiter un voilier en mer suppose de partager un espace restreint, sans possibilité de s'en échapper, et cela durant parfois plusieurs semaines: il faut compter en moyenne 3 semaines pour se rendre des Canaries aux Antilles et 4 à 5 semaines pour rejoindre Tahiti depuis Panama.

La vie familiale se déroule soit dehors, dans le cockpit, espace situé à l'arrière du voilier soit à l'intérieur, dans le carré, partie centrale du voilier. Chaque membre de la famille ne pouvant disposer de sa propre chambre, les parents choisissent le plus souvent de s'installer dans le carré et de laisser les cabines aux enfants, ces derniers devant parfois les partager avec leurs frères et sœurs. Cohabiter dans un espace restreint n'est pas toujours aisé. Les disputes au sein de la fratrie font partie des mauvais souvenirs de Sandrine qui a navigué six ans avec son frère et ses deux sœurs : « Essayez de fuir ou de vous retrouver seul sur un voilier de quatorze mètres! Il vous parait bien petit quand arrive le moment où vous ne voulez plus rien savoir de vos frères et sœurs » (Mailhot et Manny, 2003, p. 399).

En mer, la sécurité prime et réduit la liberté de mouvement des enfants. Les parents redoutent particulièrement la chute à la mer et instaurent des règles de conduite très strictes à bord: interdiction d'aller sur le pont tout seul, obligation de rester à l'intérieur du bateau en cas de mauvais temps ou de manœuvres retenant l'attention des adultes. C'est en mer que les parents expriment leur crainte et se reprochent parfois d'avoir embarqué leurs enfants dans ce périple. Lors de ses quarts de nuit, Britta s'interroge : « La nuit, je dois faire face à mes peurs et mes angoisses. Et dans ces moments là, je me demande dans quelle aventure nous avons entrainé les enfants » (Gaussen, 2003-2005).

29 Alors qu'à terre « les relations conjugales sont pensées sur un modèle égalitaire et non plus hiérarchique, la hiérarchie maritime réintroduit une hiérarchie conjugale " souligne S. Brulé-Josso (2010, p. 826). À bord d'un voilier naviguant, les relations familiales sont soumises au droit maritime qui impose la désignation d'un chef de bord, fonction dévolue au père dans tous les couples constituant notre corpus. Cette situation n'est pas spécifique aux plaisanciers au long cours et quelle que soit la durée de la navigation, une journée ou plusieurs années, «le chef de bord d'un bateau de plaisance est à plus de $90 \%$ des cas un homme " (Brulé-Josso, 2010, p. 825). Les parents "n'occupent donc pas les mêmes places dans la hiérarchie. Le rôle du chef de bord, tenu par le père, englobe celui d'équipier, occupé par la mère » (Brulé-Josso, 2010, p. 825). Au père chef de bord revient la gestion du voilier et de la navigation, à la mère 
la responsabilité des enfants et des tâches domestiques. En tant qu'équipière, elle est également amenée à participer aux manœuvres et aux quarts de nuit, qu'elle partage avec son conjoint tandis que les enfants dorment. La journée, les parents, et notamment la mère, s'occupent des enfants et ne peuvent donc se reposer... dès lors, la fatigue accumulée peut entrainer une dégradation des relations de couple à bord. Le huis clos familial dans un espace restreint, l'environnement maritime potentiellement dangereux ainsi que la hiérarchie conjugale instaurée par le droit maritime sont susceptibles de favoriser l'émergence de tensions et de conflits lors des longues navigations. La solution, majoritairement adoptée par les plaisanciers naviguant moins de trois ans, consiste à laisser le père naviguer seul tandis que la mère et les enfants prennent l'avion pour le rejoindre. Pour justifier ce choix de la séparation familiale, la raison la plus souvent invoquée est le bien-être des enfants : «Les filles n'aiment pas les grandes navigations " explique ainsi Le Gall (2003-2004). Cette séparation familiale permet également au père d'apprécier pleinement ces semaines de mer sans avoir à assumer la double fonction de chef de bord et de parent. «Le fait que pour la transat il n'y ait pas ma femme et mes filles, cela m'enlève un poids » témoigne B. Raucourt (entretien, Las Palmas). La grande croisière naît bien souvent du désir des parents de passer du temps avec leurs enfants. En escale, le voilier favorise effectivement une configuration familiale «intimiste", alors qu'il devient, lors des longues navigations, « le lieu de la scission familiale » (Brulé-Josso, 2010, p. 401).

31 L'exemple des parents plaisanciers au long cours, quantitativement marginal, fait apparaitre des traits structurants de l'expérience spatiale parentale, au-delà de la spécificité des modes de vie. Les escales de plusieurs mois doivent permettre aux parents de travailler et de scolariser leurs enfants, tout comme le choix d'un lieu de résidence permanente à terre tient compte à la fois de la proximité d'établissements scolaires répondant aux attentes des parents et du lieu de travail de ces derniers. Pour les pratiques récréatives, les escales sont choisies selon les mêmes critères que les lieux de vacances : permettre aux enfants de s'enrichir et à la famille de passer du temps ensemble tout en faisant en sorte que parents et enfants puissent être autonomes et bénéficient d'un temps qui leur soit propre.

Les parents voyageurs sont pris dans deux logiques: si le voyageur attend de l'itinérance récréative qu'elle apprenne à ses enfants la tolérance en les ouvrant à la diversité et l'autonomie, le parent est avant tout attentif à ce qu'ils ne prennent pas de retard et puissent réintégrer au retour une école traditionnelle. Dès lors la pression scolaire existe et les enfants expriment leur crainte de ne pas être au niveau. Après un voyage de six ans, Damien explique avoir « peur de revenir à l'école, peur de ne pas être à la hauteur » (Mailhot et Manny, 2003, p. 386).

33 La grande croisière familiale est souvent présentée comme idyllique dans les récits des plaisanciers, qui s'étendent rarement sur les difficultés rencontrées. La confusion des rôles, entre parents et enseignants, entre parents et chef de bord, n'est pas toujours aisée à assumer. Des échecs existent, et des familles, parties pour un tour du monde, s'arrêtent aux Canaries. Mais ceux-là ne témoignent pas. De même, ce sont le plus souvent les parents qui expriment ce qu'ils pensent être le point de vue des enfants : ils valorisent la grande croisière comme expérience marquante pour ces derniers et s'étendent peu sur les éventuelles contraintes de ce mode de vie pour un enfant. 


\section{BIBLIOGRAPHIE}

Authier J.-Y., Lehman-Frisch S., 2012. Il était une fois... des enfants dans des quartiers gentrifiés à Paris et à San Francisco. Actes de la recherche en sciences sociales, $n^{\circ}$ 195, p. 58-73.

Benson M., O’Reilly K., 2009. Migration and the Search for a Better Way of Life: A Critical Exploration of Lifestyle Migration. The Sociological Review, vol. 57, n 4, p. 608-625.

Brulé-Josso S., 2010. L'habitation du voilier de plaisance en croisière familiale. Anthropologie d'un espace et des gouvernementalités en jeu. Thèse d'ethnologie sous la dir. de J.-F. Simon, CRBC, Université de Bretagne occidentale, Brest, $1150 \mathrm{p}$.

Cohen E., 2003. Backpacking: Diversity and Change. Journal of Tourism and Cultural Change, vol. 1, $\mathrm{n}^{\circ} 2$, p. 95-110.

Cohen S.-A., Duncan T., Thulemark M. (dir.), 2016. Lifestyle Mobilities: Intersections of Travel, Leisure and Migration. Routledge, 280 p.

Cornell J., 2017. Où vont tous les bateaux ? Mouvement des voiliers dans le monde en 2015 [En ligne]. URL: https://cornellsailing.com/ Consulté en octobre 2019.

Equipe MIT, 2011. Tourismes 3, La révolution durable. Belin, 332 p.

Forget C., 2012. Vivre sur la route. Les nouveaux nomades nord-américains. Montréal, Liber, 222 p.

Gaugue A., 2020. Les plaisanciers au long cours, des habitants de mer. H.D.R. vol 1, Université Picardie Jules Verne, Amiens, 317 p.

Gaussen L. et B., 2003-2005. Journal de bord, [Blog En ligne]. URL: http://www.techfina.ch/hobbit (consulté en janvier 2007).

Gourmelen J.L., 1997. Année sabbatique. Du rêve à la réalité. Voiles et voiliers, n 315, p. 76-95.

Holloway S.-L., Plimott-Wilson H., 2014. Enriching Children, Institutionalizing Childhood? Geographies of Play, Extracurricular Activities, and Parenting in England. Annals of the Association of American Geographers, vol. 104, n 3, p. 613-627.

Korpela M., 2009. More Vibes in India. Westerners in Search of a Better Life in Varanasi. Academic Dissertation, University of Tampere, Finland, $249 \mathrm{p}$.

Korpela M., 2014. Growing up Cosmopolitan? Children of Western Lifestyle Migrants in Goa, India. In Kaunonen L., Cosmopolitanism and Transnationalism: Visions, Ethics, Practices. Helsinki Collegium for Advanced Studies, p. 90-115.

Le Bigot B., 2017. Penser les rapports aux lieux dans les mobilités privilégiées. Étude croisée des backpackers en Thailande et des hivernants au Maroc. Doctorat de géographie sous la direction de N. Cattan, Université Paris 1,559 p.

Le Gall M., 2003-2004. Journal de bord, [Blog En ligne]. URL: http://www.samos.saba.free.fr/ Consulté en mars 2005.

Maheu J.-M. et N., 1989-2002. Le voyage de Mérovée, [Blog En ligne]. URL: http://www.merovee.net (consulté en octobre 2003).

Mailhot C., Manny D., 2002. La V'limeuse autour du monde. Tome 1, édition Groupe nautique Grand Nord et Bas Saint-Laurent, Canada, 352 p. 
Mailhot C., Manny D., 2003. La V'limeuse autour du monde. Tome 2, édition Groupe nautique Grand Nord et Bas Saint-Laurent, 408 p.

Meffre M. et G., 1997. Les Meffre, deux anges au paradis. Caluire, édition du plaisancier, Caluire ; Bordeaux Loisirs nautiques, $228 \mathrm{p}$.

Mikkelsen M.V., Blichfeldt B.S., 2018. 'We have not seen the kids for hours': the case of family holidays and free-range children. In Schaenzel H., Carr N., Children, Families and Leisure. Routledge, p. 86-105.

Montbron de Y. et C., 1996-1998. Journal de bord, [Blog En ligne]. URL: http://force9.free.fr/loren (consulté en avril 2004).

Neuville de A., 1995. Le tour du monde à treize ans. Voiles et voiliers, ${ }^{\circ} 291$, p. 90-93.

Olivier et Daphné, 2010-2020 (en cours). Journal de bord, blog, http://www.altitudezero.org/ Consulté en novembre 2019.

Ortmans R. et F., 1996-2003. Journal de bord, [Blog En ligne]. URL: http://force9.free.fr/loren/farenui.htm (consulté en avril 2004).

Perret M et B., 1984. Trois p'tits mousses ... et puis s'en vont. Voiles et voiliers, $n^{\circ} 162$, p. 30-37.

Rivière C., 2017. Du domicile à la ville : étapes et espaces de l'encadrement parental des pratiques urbaines des enfants. Espaces et sociétés, $\mathrm{n}^{\circ}$ 68-169, p. 171-188.

Vacher L. 2004. Le tourisme au Kimberley et le 'big trip' des retraités australiens. Le Globe, Revue genevoise de géographie, $\mathrm{n}^{\circ}$ 144, p. 41-55.

\section{NOTES}

1. «la mobilité d'individus privilégiés de tout âge, qui, temporairement ou définitivement, migrent vers des lieux qui pour diverses raisons, leur offrent une meilleure qualité de vie».

2. « déplacements récurrents et semi-permanents de durée variable».

3. URL : https://lfadm.jimdofree.com, consulté en octobre 2020.

4. Ces blogs sont cités par le(s) nom(s) de leur(s) auteur(s) suivi(s) des dates de leur grande croisière (voir en Bibliographie).

5. «Ce n'est pas la naissance d'un enfant mais bien le moment où celui-ci entre à l'école qui constitue un moment crucial pour décider de quitter Vârânasî ou d'y rester ».

6. «Les parents ont souvent souligné que le fait de vivre là-bas était bénéfique pour leurs enfants, beaucoup d'entre eux semblant expliquer leur mode de vie en affirmant que c'était mieux pour leurs enfants ".

7. «Dans le cas des migrations d'agrément, les familles socialisent rarement avec les familles indiennes locales». Dès lors, « les enfants ne sont pas sociabilisés dans le cadre culturel local mais selon un mode de vie occidental ».

8. Best F., 1979. Education nouvelle. Courants pédagogiques actuels: problèmes idéologiques et philosophiques, cité par Brulé-Josso, 2010, p. 979.

9. «Transporter les enfants d'une activité à l'autre est, pour les parents, une lourde charge de travail.».

10. « des vacances réussies permettent de s'affranchir des obligations familiales pour que tous les membres de la famille puissent profiter de leurs vacances, ensemble ou séparément ». 


\section{RÉSUMÉS}

Cet article porte sur la géographie des parents voyageurs à travers l'exemple des plaisanciers au long cours, ceux qui durant au moins une année et souvent bien davantage ont navigué d'escale en escale avec leurs enfants. L'ouverture sur le monde et le repli sur la famille sont les principales motivations avancées par les parents pour expliquer leur souhait de naviguer avec leurs enfants. Au cours de l'itinérance, les parents plaisanciers adaptent le choix des lieux d'escales et leur durée à ce qu'ils considèrent comme essentiel pour leurs enfants : leur scolarité et la possibilité d'être en contact avec d'autres enfants de leur âge. Des espaces océaniques aux ilots tropicaux, l'encadrement parental varie selon les lieux fréquentés.

This article looks at the geography of seafaring parents travelling with their children from port of call to port of call for at least a year, and often much longer. To explain their choice of sailing with their children, the parents often put forward an openness to the world and a turning inward to the family as the main motivations. During the cruise, these parents on the move choose the length and location of the stopovers according to what they consider as essential for their children: their schooling and the opportunity to be in contact with other children. Be it oceanic areas or tropical islets, parental supervision also varies according to the place frequented.

\section{INDEX}

Mots-clés : voyage, plaisance, parent, migration d'agrément, mode de vie mobilitaire

Keywords : Travel, ocean cruising, parent, life style migration, life styles mobility

Thèmes : Sur le Champ - Sur le Terrain

\section{AUTEUR}

\section{ANNE GAUGUE}

Gaugue Anne, anne.gaugue@wanadoo.fr, est maître de conférences à l'Université Clermont Auvergne et membre de l'équipe CHSSC, Université Picardie Jules Verne. Elle a récemment publié :

- Gaugue Anne, 2018. Les plaisanciers au long cours. Des voyageurs pas comme les autres. Espaces, $n^{\circ} 343$, p. 28-34.

- Gaugue Anne, 2017. S'amuser sur la mer. Les espaces du nautisme. In Fagnoni E. (dir.), Les espaces du tourisme et des loisirs. Paris, Armand Colin, p. 220-233.

- Gaugue Anne, 2017. Lieux centraux et périphériques dans les itinéraires touristiques : l'exemple des croisières hauturières. In Bernard N., Blondy C., Duhamel Ph. (dir.), Tourisme, marge et périphérie. Presses Universitaire de Rennes. 